\title{
L'INTÉGRATION D'INDICES PRONOMINAUX AU MOT VERBAL: UN ESSAI DE TYPOLOGIE
}

\section{INTRODUCTION.}

Les travaux de Tesnière préfigurent sur plus d'un point les recherches en typologie syntaxique tels qu'ils se sont développés depuis une vingtaine d'années, et qui souvent n'ont fait que confirmer et préciser des hypothèses déjà formulées dans les Éléments de Syntaxe Structurale. On sait ce qu'il en est pour l'étude typologique de l'ordre des constituants de diverses constructions, qui depuis Greenberg a connu un développement spectuculaire. Mais on peut aussi trouver exposée chez Tesnière (Éléments de Syntaxe Structurale, chapitres 59 à 62) la notion d'indice qui, convenablement précisée, constitue la base indispensable d'un autre aspect fondamental de la typologie syntaxique: la présence à l'intérieur du mot verbal de morphèmes dont la fonction est de représenter des noms occupant une fonction déterminée relativement au prédicat verbal. Il manquait toutefois à Tesnière une définition précise, explicite et opératoire de la notion de constituant nominal pour tirer toutes les conséquences de la reconnaissance de ce type d'unité.

\section{LA NOTION D'INDICE.}

L'idée fondamentale d'où découle la notion d'indice est que les descriptions d'un nombre important de langues (en premier lieu le français) proposent une délimitation de l'ensemble des «pronoms personnels» qui doit être révisée, car cet ensemble regroupe deux types d'unités très différents du point de vue morphosyntaxique:

- Des unités comme moi, toi, lui ont une distribution qui autorise à les reconnaître syntaxiquement comme noms.

- Des unités comme je, $\underline{\text { tu}}, \underline{\mathrm{il}}$ ont une distribution tout à fait spécifique: même si cela n'est pas évident lorsqu'on se limite à observer des phrases minimales, on peut facilement montrer que ces unités occupent dans la construction de la phrase des positions où elles ne commutent pas avec les noms qu'elles preprésentent. Par exemple:

$\begin{array}{rlll}\text { Michel } & \text { aussi } & \text { (il) } & \text { chante } \\ \text { lui } & \text { aussi } & \text { (il) } & \text { chante } \\ \text { moi } & \text { aussi } & \text { je } & \text { chante } \\ \text { toi } & \text { aussi } & \text { tu } & \text { chantes }\end{array}$


D'autre observations allant dans le même sens montrent que je, 뜨, lㅣ présentent par rapport au verbe une absence d'autonomie telle qu'il convient de considérer que ces morphèmes sont, non pas des mots à part, mais des formants du mot verbal. En particulier, ils ne se prêtent pas à la coordination. Autrement dit, du point de vue dù fonctionnement actuel du français, il n'y a pas de différence fondamentale entre ces morphèmes et ceux traditionnellement identifiés comme «désinences personnelles du verbe».

On peut donc réunir sous le terme d'indice pronominal (en abrégé: indice) tous les morphèmes qui représentent des expressions nominales tout en occupant des positions syntaxiques qui n'autorisent pas à les identifier comme constituants nominaux. Cette catégorie englobe, à côté des «désinences personnelles du verbe», une partie des unités traditionnellement identifiées comme «pronom personnel». En effet, l'exemple du français montre que souvent se trouvent réunis sous cette dénomination à la fois des noms déictiques qui ont les mêmes propriétés syntaxiques que les expressions nominales qu'ils représentent (ㅉoi, toi, $\underline{\text { lui) }}$ ) et des unités qui historiquement sont d'anciens noms dictiques mais qui ont pris dans l'évolution de la langue un statut syntaxique différent de celui des expressions nominales qu'ils représentent.

\section{LE NOMBRE D'INDICES PRONOMINAUX INTÉGRÉS AU MOT VERBAL.}

L'existence de morphèmes appartenant au mot verbal et représentant un des constituants nominaux reliés au verbe en tant que prédicat n'a rien d'universel: dans un certain nombre de langues (japonais, mandingue, etc.), toutes les unités déictiques ont syntaxiquement le statut de nom. Toutefois, une fois admis qu'une bonne partie des unités traditionnellement reconnues comme «pronoms personnels» sont en réalité des indices pronominaux affixés à la base verbale, il apparaît que ce phénomène est beaucoup plus répandu et développé qu'on ne le pense généralement.

Dans beaucoup de langues, le mot verbal se caractérise par l'intégration d'un unique indice pronominal. C'est le cas du latin et des autres langues indo-européennes anciennes, mais c'est aussi le cas de nombreuses langues à travers diverses familles génétiques: turc, finnois, etc..

Le cas de langues où le mot verbal peut intégrer (en fonction de la valence du verbe) deux indices pronominaux est très répandu aussi. On peut citer le hongrois, le quiché (maya), le nahuatl.

Enfin, le cas de langues où le mot verbal peut intégrer plus de deux indices pronominaux peut être illustré par exemple par le tswana (bantou): dans cette langue, kiálómònósédítsè «je le lui ai donné à boire pour elle» constitue un mot unique où kì est l'indice d'élocutif singulier et où áa, $\sim 1 \hat{\omega} \sim$ et $\simeq$ mò $\sim$ sont des indices de délocutif représentant respectivement màfî «lait», lòsíá «bébé» et mòsádí «femme». Une fois montré que les «pronoms personnels conjoints» du français sont en fait des indices pronominaux faisant morphologiquement partie du mot verbal, on doit reconnaître que cette situation est aussi celle du français: dans une stricte analyse synchronique, je le lui 
dirai constitue un mot unique incorporant à son initiale trois indices pronominaux successifs.

\section{LE CONDITIONNEMENT DE L'EMPLOI DES INDICES PRONOMINAUX.}

Dans certaines langues, la présence d'un indice pronominal est strictement limitée au cas où l'expression nominale qu'il représente a du point de vue discursif le statut d'élément thématique. Cette situation est illustrée de façon typique par le tswana. Mais la présence d'un indice pronominal peut constituer une pure contrainte morphosyntaxique ne faisant intervenir en rien le statut discursif de l'expression nominale qu'ils représentent. C'est notamment le cas de l'indice pronominal intégré à la désinence du verbe latin.

On peut considérer comme une situation intermédiaire le cas (fréquent) où l'emploi d'un indice pronominal n'est obligatoire qu'en relation avec des expressions nominales marquées comme définies. En effet, «défini» fait référence à des caractéristiques intrinsèques de l'expression nominale, mais cette notion a néanmoins une relation évidente avec les fonctions discursives: une expression nominale définie n'est pas nécessairement thématique, mais elle a tout de même une certaine prédisposition à l'être.

\section{LES DISTINCTIONS QUI INTERVIENNENT DANS LE CHOIX DES INDICES PRONOMINAUX.}

Le choix des indices pronominaux tient très généralement compte du statut énonciatif de leur référent (élocutif / allocutif / délocutif) et du nombre (singulier/ pluriel, plus rarement singulier / duel / pluriel). D'autres distinctions peuvent se manifester, le plus souvent au délocutif (le tswana par exemple offre le choix entre 11 indices de délocutif selon la classe d'accord à laquelle appartient l'expression nominale représentée), mais ceci n'est pas exclusif: dans un certain nombre de langues (l'arabe, le hausa) la distinction masculin / féminin s'étend aux indices d'allocutif.

Il est intéressant de remarquer que parfois, l'indice pronominal reflète de façon obligatoire des distinctions qui ne sont pas nécessairement explicitées au niveau de l'expression nominale qu'il représente. C'est par exemple le cas de la distinction singulier / pluriel en quiché. Ceci doit inciter à approfondir le réflexion sur la notion d'«accord», qu'il n'est pas possible de concevoir comme étant la simple copie de caractéristiques d'un terme de la phrase sur un autre.

\section{LE DEGRÉ D'INTÉGRATION MORPHOPHONOLOGIQUE DES INDICES PRONOMINAUX.}

Dans leur forme, les indices pronominaux peuvent se différencier plus ou moins nettement des noms déictiques auxquels ils correspondent. Ainsi en français, l'indice je n'a aucune ressemblance formelle avec le nom déictique moi, tandis que l'indice elle est entièrement homonyme du nom déictique elle. 
Le degré d'intégration morphophonologique des indices pronominaux au mot verbal est variable selon les langues: à côté de cas où il n'y a aucune difficulté à découper les formes verbales en segments successifs dont certains s'identifient comme indices pronominaux (cf. l'exemple tswana cité ci-dessus: kì-á-1ó-mò-nósédítsè), il y en a d'autres où les indices pronominaux donnent lieu à des amalgames plus ou moins poussés avec d'autres formants du mot verbal (situation qui peut être illustrée par les désinences verbales des langues indo-européennes). Lorsque le verbe incorpore plusieurs indices pronominaux, ils peuvent fusionner entre eux. Un cas extrême est celui du hongrois: dans les désinences verbales de la «conjugaison objectale» de cette langue, il s'avère impossible de séparer l'indice de sujet et l'indice d'objet comme deux segments distincts.

\section{CONCLUSION: ASPECTS DIACHRONIQUES DE LA QUESTION.}

Au vu des données disponibles, il est raisonnable d'admettre que, dans les langues où ils existent, les indices pronominaux incorporés au mot verbal sont d'anciens noms déictiques qui au cours de l'évolution se sont trouvés en quelque sorte satellisés par le mot verbal, relativement auquel ils ont perdu toute autonomie. De telles évolutions peuvent d'ailleurs se trouver compensées par d'autres évolutions, elles aussi manifestement très communes, qui aboutissent à créer de nouveaux noms déictiques par le figement d'anciens syntagmes nominaux de sens déictique (ce qui explique en particulier pourquoi, dans les «pronoms personnels» de bien des langues, on peut reconnaître plus ou moins nettement des morphèmes identifiés par ailleurs comme «possessifs»).

Une fois satellisés par le verbe, les anciens noms déictiques devenus des indices pronominaux s'engagent parallèlement dans deux évolutions logiquement indépendantes mais qui dans les faits semblent aller très généralement de pair:

- Du point de vue syntaxique, les indices pronominaux au premier stade de leur évolution ne s'emploient que pour représenter des éléments thématiques (dont l'indice constitue souvent la seule trace dans l'énoncé réalisé); dans un deuxième stade, ils tendent à s'employer de manière obligatoire pour représenter divers types de constituants nominaux prédisposés à avoir un statut thématique (noms propres, syntagmes à déterminant déictique) même dans le cas où les constituants nominaux en question n'ont pas ce statut. Au terme de cette évolution, leur emploi relève de pures règles morphosyntaxiques $\mathrm{d}$ 'accord dans lesquelles n'intervient en rien le statut discursif de l'expression nominale qu'ils représentent.

- Du point de vue morphophonologique, les indices pronominaux incorporés au mot verbal tendent à subir les évolutions caractéristiques des formants d'un mot, c'est à dire que leur frontière avec les autres formants du mot verbal tend à donner lieu à des processus phonologiques pouvant conduire à une fusion plus ou moins poussée.

Les langues indo-européennes sous leur forme la plus anciennement attestée présentent un verbe incorporant dans sa désinence un indice pronominal et un seul, et 
cet indice pronominal se trouve manifestement au stade final des évolutions esquissées ci-dessus - d'où d'ailleurs la difficulté, bien connue des indo-européanistes, à reconstituer l'origine des «désinences personnelles» du verbe indo-européen en les rapprochant des «pronoms» correspondants. Du point de vue diachronique, on peut imaginer deux directions d'évolution possibles, qui sont effectivement toutes deux attestées par les évolutions récentes des langues indo-européennes:

- disparition de l'indice pronominal ancien, du fait d'une tendance assez générale des langues à éliminer des morphèmes parvenus à un certain stade de complexité morphophonologique et d'amalgame;

- création d'indices pronominaux nouveaux par satellisation d'unités qui, dans l'état le plus anciennement connu de ces langues, avaient le statut de noms déictiques.

La comparaison des langues romanes et des langues germaniques est de ce point de vue intéressante. En effet, les langues germaniques ont plus ou moins bien conservé l'indice de sujet désinenciel hérité de l'indo-européen, mais ces langues ne semblent pas avoir connu les évolutions qui auraient pu aboutir à la création de nouveaux indices à partir d'anciens noms déictiques. Dans les langues romanes par contre, la tendance générale est à maintenir l'indice de sujet hérité de l'indo-européen et à créer de nouveaux indices représentant les constituants nominaux en fonction autre que sujet. Dans les langues romanes autres que le français, les «pronoms personnels sujets» restent bien des formes nominales autonomes, tandis que les «pronoms personnels compléments» ne restent des formes nominales autonomes que pour une partie d'entre eux, les autres s'incorporant au mot verbal (ce qu'enregistrent de manière variable les orthographes des langues romanes). La même tendance caractérise les langues balkaniques dans leur ensemble, quelle que soit la famille à laquelle elles appartiennent.

Mais parmi les langues ayant ainsi développé des indices pronominaux susceptibles de représenter des constituants nominaux de fonctions syntaxiques diverses, le français représente à l'échelle des langues indo-européennes d'Europe un cas absolument unique. En effet, comme les autres langues romanes le français a développé un système d'indices pronominaux intégrés au mot verbal et susceptibles de représenter des expressions nominales en fonction autre que sujet (me, te, le/la/lui, nous, vous, les/leur). Mais de plus, à la différence des autres langues romanes où l'indice de sujet désinenciel a été maintenu, il y a en français une tendance très forte (même si elle n'a pas encore tout à fait abouti) à la disparition de l'indice de sujet désinenciel, tendance dont la contrepartie est l'instauration de nouveaux indices de sujet, préfixés au verbe. Ainsi, le verbe français, dans sa structure morphologique, s'est considérablement écarté du type représenté par les langues indo-européennes anciennes pour se rapprocher d'un type caractérisé par la préfixation à la base verbale de plusieurs indices successifs susceptibles de représenter divers constituants nominaux reliés au verbe, et pas seulement le sujet. 


\section{Povzetek}

\section{SPAJANJE ZAIMKOVNIH PRVIN V GLAGOL: POSKUS TIPOLOGIJE}

Tesnière posveča poglavja od 59 do 62 svojih Elementov strukturalne skladnje posebni vrsti morfemov, za katere se tukaj predlaga ime indices pronominaux, zaimkovne skladenjsko-oblikovne enote, in jih definira (pri Tesnièrju je definicija nekako ohlapna) kot morfeme, ki so samostalniškim enakovrednii, vendar pa v zgradbi stavka zasedajo mesta, kjer niso izmenljivi s samostalniškimi izrazi, ki jih sicer lahko nadomeščajo. Ta definicija zaobsega končnice osebnih glagolskih oblik, pa tudi del enot, ki jih po tradiciji imenujemo "osebne zaimke". Če to že ni zmeraj tako, pa delujejo te skladenjsko-oblikovne enote $v$ glavnem vendar kot glagolski formanti.

Za tipologijo spajanja teh zaimkovnih prvin $v$ glagolske lahko porabimo tale merila:

1. Število zaimkovnih prvin povedka, ki se v kakem jeziku pojavljajo: nič, ena, dve, več kot dve.

2. Skladenjsko in/ali pragmatično odvisnost rabe teh prvin, ki natančneje določajo vrednosti povedka; "zaimki" se po tradicionalni klasifikaciji lahko ravnajo po samostalniškem izrazu, na katerega se povedek nanaša in ki je prevzel vlogo téme; vendar pa je raba zaimkovne prvine $v$ nekaterih jezikih zgolj oblikoslovno-skladenjska prisila; zamišljamo pa si lahko tudi vse mogoče vmesne stopnje.

3. Pomenske značilnosti odnosnice, vidne v izbiri zaimkovne enote (oseba, število, spol, npr. pri vljudnem naslavljanju).

4. Stopnja oblikoglasne zlitosti z glagolskim leksemom.

Značilnosti zaimkovnih prvin kažejo vključenost diahronije v sinhroni opis nekega jezika. 